\title{
Estrategias Proyectuales para un Hábitat Emergente e Inclusivo Sistema Incremental Modular para Situaciones Habitacionales
}

\section{Project Strategies for an Emerging and Inclusive Habitat Modular Incremental System for Housing Situations}

\author{
Dr. Arq. Juan Carlos Etulain, Esp. Arq. Alejandro Lancioni, Arq. Gabriel Ignacio Di Gilio, \\ Arq. Luciano Di Gregorio Luchetti. Estudiantes: Benjamín Saab, Nicolás Luna Weber \\ Facultad de Arquitectura y Urbanismo (FAU) \\ Universidad Nacional de La Plata (UNLP) \\ etulain.lancioni@gmail.com - jcetulain@fau.unlp.edu.ar
}

\section{RESUMEN}

El trabajo presenta propuestas que procuran atender el acuciante déficit habitacional a nivel nacional, así como las urgencias generadas por desastres socio-naturales, dando respuesta a las necesidades emergentes de los sectores más desprotegidos y vulnerables, a partir de explorar y formular soluciones habitacionales modulares sustentables de bajo costo, mediante la utilización de sistemas abiertos y adaptativos prefabricados / industrializados con distintas materialidades. Se parte de la hipótesis que actuar para el hábitat de la emergencia, con un enfoque inclusivo, requiere desde lo proyectual imaginación en pos de lograr eficiencia y síntesis creativa superadora de lo existente. Se utilizó una estrategia exploratoria a partir de identificar referentes nacionales e internacionales, utilizando técnicas e instrumentos propios de la investigación proyectual. En este marco, se presentan dos experiencias, una para habitar temporalmente y otra un Sistema Incremental Modular (SIM), que tiende a dar respuesta a sectores vulnerables a partir de distintas configuraciones espaciales.

\section{ABSTRACT}

The work presents proposals that seek to address the pressing housing deficit at the national level, as well as the emergencies generated by socio-natural disasters, responding to the emerging needs of the most unprotected and vulnerable sectors, from exploring and formulating sustainable modular housing solutions Low cost, through the use of open and adaptive prefabricated / industrialized systems with different materialities. It is based on the hypothesis that acting for the emergency habitat, with an inclusive approach, requires imagination from the project point of view in order to achieve efficiency and creative synthesis that surpasses what already exists. An exploratory strategy was used by identifying national and international references, using techniques and instruments typical of project research. In this framework, two experiences are presented, one to temporarily inhabit and the other a Modular Incremental System (SIM), which tends to respond to vulnerable sectors based on different spatial configurations.

PALABRAS CLAVE: Vivienda Transportable - Configuraciones espaciales - Sistemas abiertos y adaptativos - prefabricación - industrialización

KEY WORDS: Transportable housing - Spatial configurations - Open and adaptive systems prefabrication - industrialization

FECHA DE RECEPCIÓN: 26/8/2021 | FECHA DE ACEPTACIÓN: 8/11/2021

DOI: http://dx.doi.org/10.30972/arq.0185672 


\section{INTRODUCCIÓN}

El trabajo presenta propuestas que procuran atender el acuciante déficit habitacional existente a nivel nacional (aprox. 3,5 millones de vivienda), así como las urgencias generadas por distintos desastres socio-naturales, dando respuesta a las necesidades emergentes de los sectores más desprotegidos y vulnerables de la sociedad, a partir de explorar y formular soluciones habitacionales modulares y sustentables de bajo costo, mediante la utilización de sistemas abiertos y adaptativos prefabricados / industrializados con distintas materialidades: Acero, Madera y Hormigón, que sean apropiados a diversos contextos.

Desde esta perspectiva, Hábitat según la real academia es un conjunto de factores físicos y geográficos que inciden en el desarrollo de un individuo, una población, una especie o grupo de especies determinados (...) entorno o conjunto de factores geográficos relativos a la vida del hombre. Mientras que Emergencia, asunto o situación imprevista que requieren una especial atención y deben solucionarse lo antes posible.

En este marco, se entiende -a manera de hipótesis- que actuar en la emergencia o para el Hábitat Emergente y/o vulnerable con un enfoque inclusivo, requiere desde lo proyectual, imaginación en pos de alcanzar la eficiencia y síntesis creativa superadora de lo existente, operativizando y dando respuesta a conceptos como:

- Transitorio, que se habita mientras dure la emergencia;

- Liviano, menor peso, menos energía y fácil traslado;

- Transportable, trasladable por algún medio de transporte terrestre, aéreo o acuático y con un alto metraje cubico;

- Desmontable, con posibilidad de volver a montar con facilidad en otro lugar (opcional según la emergencia);

- Confortable, espacialmente, higrotérmicamente y funcionalmente;

- Materiales locales, reciclables y sustentables de existencia en la región;

- Respuesta rápida, fácilmente trasladable, montable y desmontable;

- Producción en serie y en fábrica, entregando el producto terminado en el lugar; y finalmente,

- Estoqueable, guardable en depósitos ocupando la menor superficie posible (opcional según la emergencia).

Metodológicamente, se utilizó una estrategia exploratoria a partir de identificar referentes nacionales e internacionales, utilizando técnicas e instrumentos propios de la investigación proyectual.

\section{FUNDAMENTACIÓN}

Las consecuencias del aislamiento social en el que se sumergió el mundo para combatir el Covid19 dejó en claro algo: que el planeta necesita un respiro. Esto se suma al problema principal que enfrenta el mundo como es el Cambio Climático, el cual va dejando sus consecuencias a partir de distintos desastres socio-naturales como inundaciones, tormentas, terremotos, sequias, incendios forestales, actividad volcánica, entre otros, eventos que ven acortado su periodo de recurrencia por incremento del Cambio Climático a escala global.

Por lo tanto, luego de una catástrofe o en condiciones de pandemia, un refugio o un módulo de emergencia ante una contingencia del habitar se convierte en un abrigo, que permite conformar un ámbito transitorio para la familia y mejorar la resiliencia ante el acontecimiento sufrido, pensando que en un futuro pueda conformar una solución habitacional de carácter estable bajo el concepto de diseño incremental.

Pero también existe otro universo de población vulnerable a que hay que dar respuestas. El incremento de los asentamientos informales (4.400 en el país, al 27-9-2020) ha sido exponencial en los últimos años, y constituyen verdaderos espacios de desigualdad y segregación social al 
que debe atenderse con responsabilidad y urgencia, brindándoles un hábitat digno e inclusivo, partiendo de una solución habitacional que dignifique a sus moradores, incorpore infraestructura, servicios y equipamientos colectivos, con actividades productivas no contaminantes basadas en la naturaleza y no en contra de ella.

Otro grupo vulnerable -cada vez mayoritario- es el de ciudadanos jóvenes que necesitan acceder a su primera vivienda en un mercado inmobiliario dolarizado y en alza en cuanto a las cotizaciones, a lo que se suma, salarios en baja o empleos informales que conlleva la imposibilidad de acceso (cuando no ausencia) a un crédito hipotecario. Finalmente, en un país donde se estima que el efecto pandemia llevara al 50\% la población por debajo del nivel de pobreza (incrementándola en un 15\%), confinándola a vivir en condiciones de marginalidad y hábitat deficitario, se entiende que la vulnerabilidad social se expresa en sus más diversas modalidades y se ve como urgente y prioritario atender esta problemática.

Por lo tanto, el momento actual es una gran oportunidad para replantearse el uso de los recursos naturales y empezar a construir con responsabilidad, una realidad más amigable con la naturaleza. En un planeta que busca la eficiencia energética y la sustentabilidad, el sector de la construcción no puede ni debe estar ausente, por lo tanto, el uso inteligente de los recursos es fundamental en un proyecto arquitectónico. Sin lugar a dudas, de las crisis nacen oportunidades, y este es el momento para repensar y desarrollar nuevos modos de producir y construir, reduciendo la huella ambiental en el planeta sin dejar de lado el confort y mejoramiento de la calidad de vida de las personas que lo habitan.

\section{ESTRATEGIAS PROYECTUALES}

El artículo desarrolla dos experiencias proyectuales realizadas para distintas convocatorias del ámbito nacional durante el año 2020. La primera, relacionada con la formulación de un prototipo para atender emergencias producto de distintos eventos peligrosos, para habitar temporalmente. La misma fue realizada para distintas regiones bioclimáticas del país: Región Patagonia - Centro y Cuyo y NEA - Pampeana. La segunda, tuvo por objetivo desarrollar un sistema incremental modular que permita dar respuestas a las necesidades de soluciones habitacionales de sectores vulnerables del país, a partir de distintas configuraciones espaciales.

La formulación para la emergencia, fue desarrollado a escala de conjunto, para tres localizaciones: urbana, periurbana y rural, de distintas ciudades expuestas a eventos peligrosos; y del prototipo. A nivel del conjunto, como estrategia de flexibilidad y replicabilidad se prevé el agrupamiento de 6 prototipos para distintos habitantes como unidad de convivencia mínima, que incorpora un módulo de infraestructura. En cuando a la sustentabilidad, el agrupamiento y las implantaciones propuestas contemplan los aspectos climáticos (orientaciones y vientos predominantes) y geomorfológicos de la región para adecuarse a la emergencia abordada.

La lógica proyectual utilizada a nivel del prototipo se basa en dar respuesta a las emergencias a partir de los conceptos citados dentro de la idea de vivienda refugio o galería según las características de las regiones, partiendo de un módulo base mínimo desplegable que mediante el sistema modular plegable plug in o conectable, permite el crecimiento bajo la lógica de la incorporación de un módulo de uso que incrementa la capacidad de habitantes (4 a 6 personas) de la vivienda mínima del módulo base inicial (1 a 2 personas). (Ver Figura 1 y 2 ) 
Estrategias Proyectuales para un Hábitat Emergente e Inclusivo Sistema Incremental Modular para Situaciones Habitacionales

Etulain, Lancioni, Di Gilio, Di Gregorio Luchetti. Estudiantes: Saab, Weber

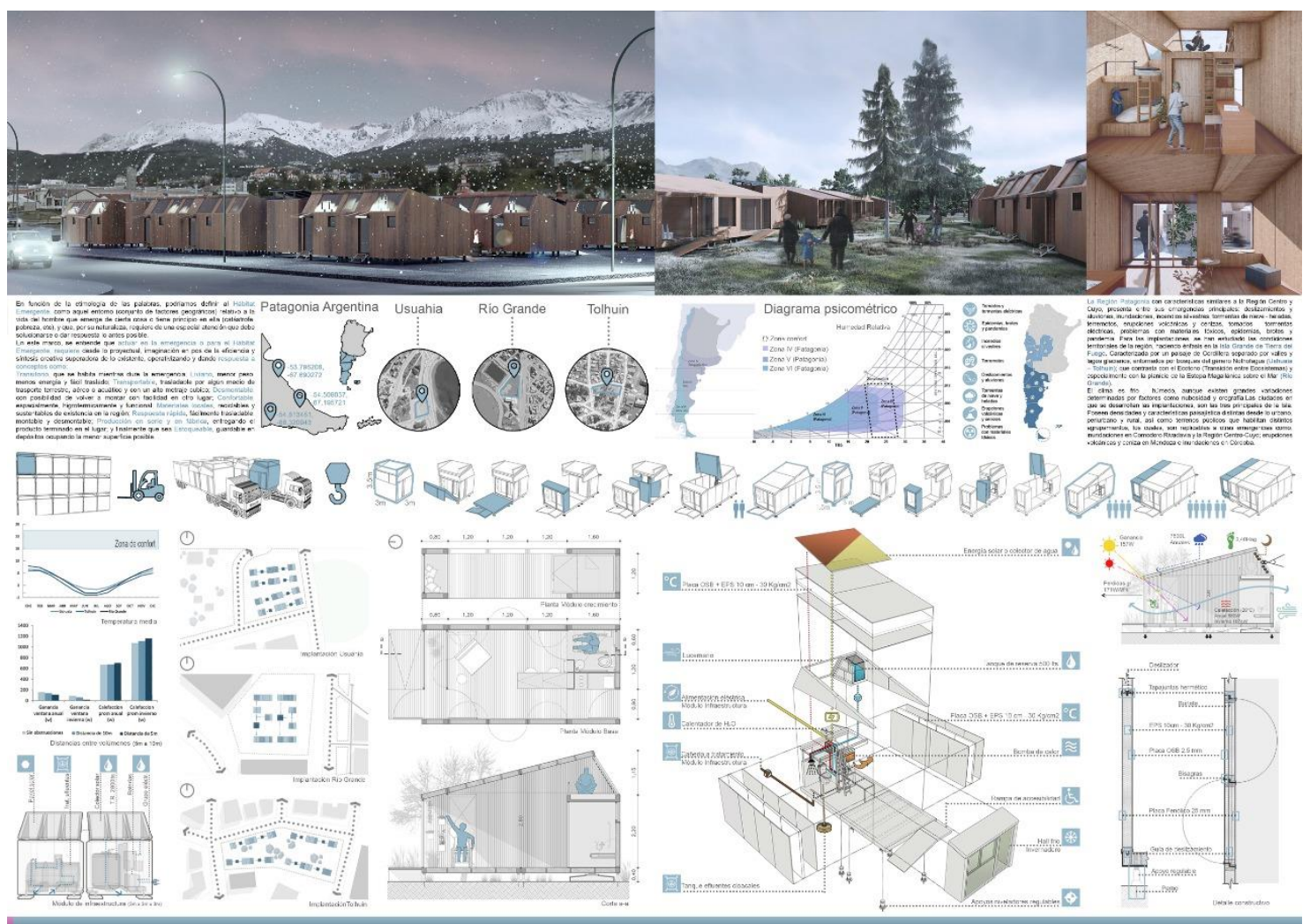

Figura 1: Prototipo de Emergencia Regiones Patagonia-Centro y Cuyo. Argentina. Fuente: Elaboración Propia

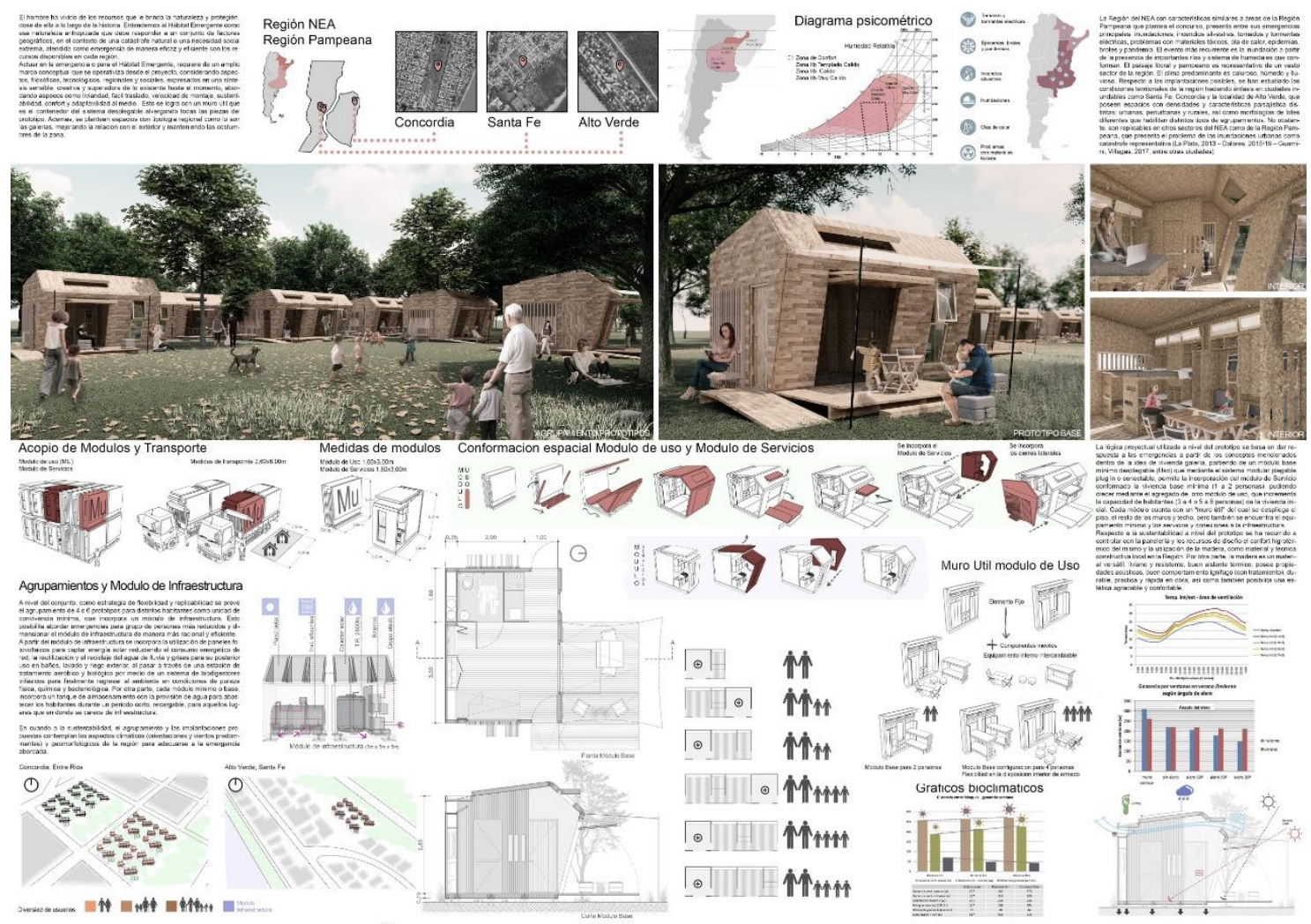

Figura 2. Prototipo de Emergencia Regiones NEA - Pampeana. Argentina. Fuente: Elaboración propia 
Respecto a la sustentabilidad a nivel del prototipo se ha recurrido a controlar con la panelería y los recursos de diseño, el confort higrotermico del mismo. La utilización de la madera, como material y técnica constructiva local en las regiones desarrolladas, además de ser versátil, liviano y resistente, buen aislante térmico, posee propiedades acústicas, buen comportamiento ignifugo (con tratamiento), durable, practico y rápido en obra, así como también posibilita una estética agradable y confortable.

A partir del módulo de infraestructura se incorpora la utilización de paneles fotovoltaicos para captar energía solar reduciendo el consumo energético de red o generada por un grupo electrógeno; la reutilización y el reciclaje del agua de lluvia y grises para su posterior uso en baños, lavado y riego exterior, al pasar a través de una estación de tratamiento aeróbico y biológico por medio de un sistema de biodigestores para finalmente regresar al ambiente en condiciones de pureza física, química y bacteriológica. Por otra parte, cada módulo mínimo o base del prototipo, incorpora un tanque para agua gris (reciclable) y otro de almacenamiento (bebible), con la provisión de agua para abastecer los habitantes durante un periodo corto (recargable), en lugares donde se carece de servicios e infraestructura.

La formulación del Sistema Incremental Modular (SIM), es una propuesta que se sustenta en una exploración proyectual, tecnológica y su proceso de producción articulado con la industria nacional o con las capacidades industriales, basado en la generación de un sistema abierto y adaptativo, que no pretende dar una respuesta acabada. Una arquitectura centrada en la indeterminación como estrategia proyectual y en una lógica sistemática de módulos funcionales con distintas materialidades: Acero, Madera y Hormigón, que permiten elegir la composición de elementos que mejor se adapten a cada contexto: urbano, suburbano o rural; necesidad: emergencia, refugio, vivienda unifamiliar; Región: bioclimática y cultural y de usuario: posibilitando un diseño personalizado e incremental atendiendo a los destinatarios de la vivienda.

Soluciones habitacionales (SH) que conforman espacios para vivir, para trabajar... una casa, un refugio, un estudio; en el campo, en la playa, en el bosque, en la montaña, en la ciudad, que requieren una evaluación de las condiciones del lugar donde se implantará el proyecto para definir la mejor opción. (Ver Figuras 3 a 4)

Esta estrategia está pensada más como un método y solo existe en virtud de su capacidad de cambio y adaptación. SIM-SH incorpora conceptos contemporáneos desde lo organizativo, funcional y tecnológico. Pretende ser una alternativa a la construcción tradicional, incorporando todas las ventajas que la industria del prefabricado puede brindar: precisión, rapidez, menor cantidad de generación de desperdicios y, sobre todo, una mayor responsabilidad ambiental.

La incorporación de la noción plug\&amp (conexión, montaje y ampliación); tiene como posibilidad y propósito deseable producir soluciones habitacionales dentro de la industria 4.0, en máquinas automáticas controladas por computadora que garanticen las condiciones necesarias para producir con calidad, trazabilidad y residuos tendientes a cero.

Pensado para generar una respuesta rápida, económica y de fácil puesta en práctica, constituye una forma innovadora de construcción, donde prima la calidad, por el proceso constructivo regulado y realizado en fábrica. Este aspecto, provee un mayor grado de precisión en la ejecución de las soluciones habitacionales, para luego ser transportada al sitio donde se montará la configuración espacial adoptada por el usuario o sector del Estado que implemente la propuesta.

El 90 por ciento de la obra se realiza en una planta industrial. El $10 \%$ restante se ejecuta en el lugar de montaje y tiene que ver con las fundaciones, instalaciones de la vivienda a los servicios públicos, agua, luz y la cámara séptica. Los tiempos de construcción son muy rápidos. Los movimientos y tiempos de construcción son mínimos. 
Estrategias Proyectuales para un Hábitat Emergente e Inclusivo

Sistema Incremental Modular para Situaciones Habitacionales

Etulain, Lancioni, Di Gilio, Di Gregorio Luchetti. Estudiantes: Saab, Weber

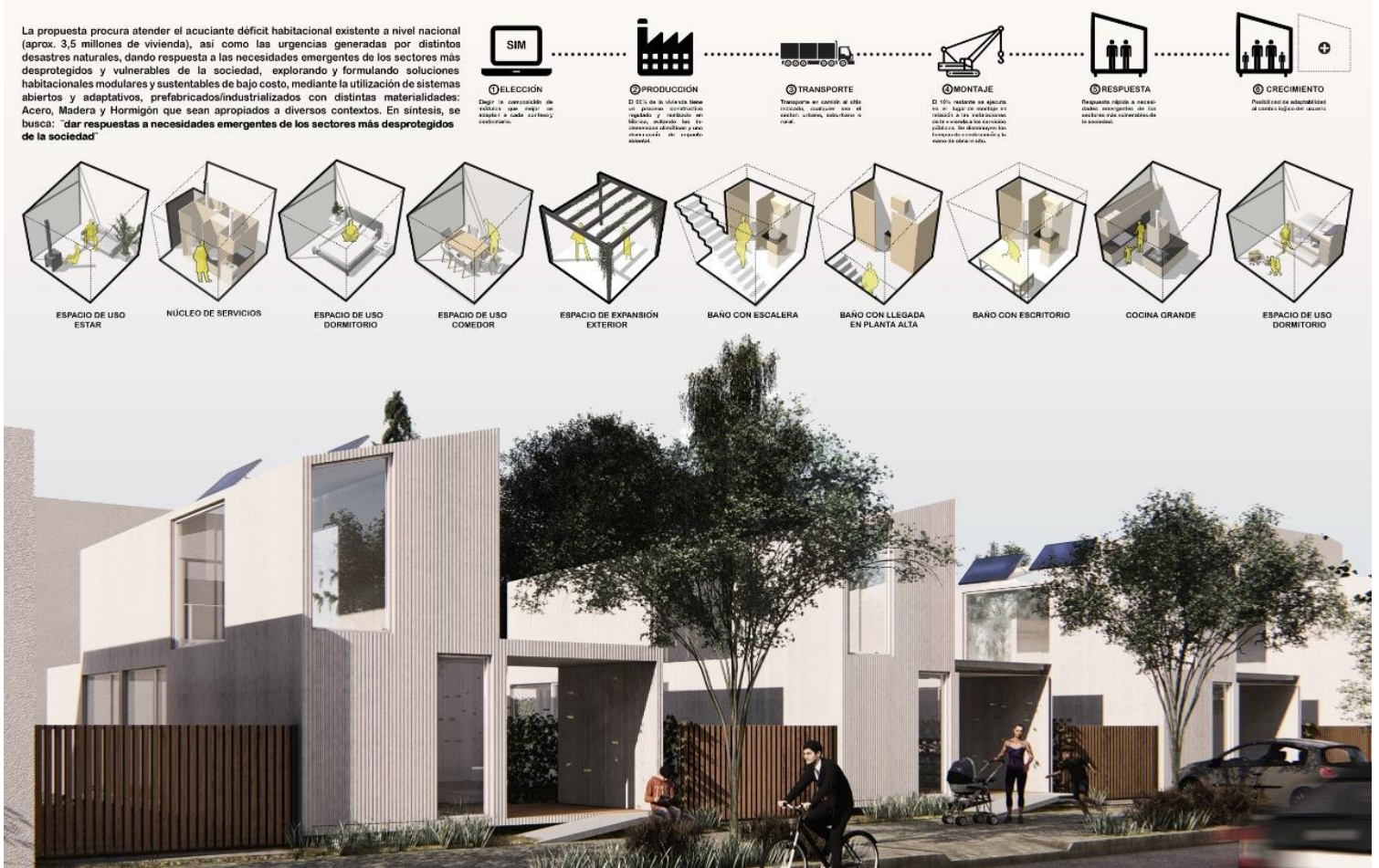

Figura 3: Sistema Incremental Modular. Proceso de Producción y Módulos. Fuente: Elaboración Propia

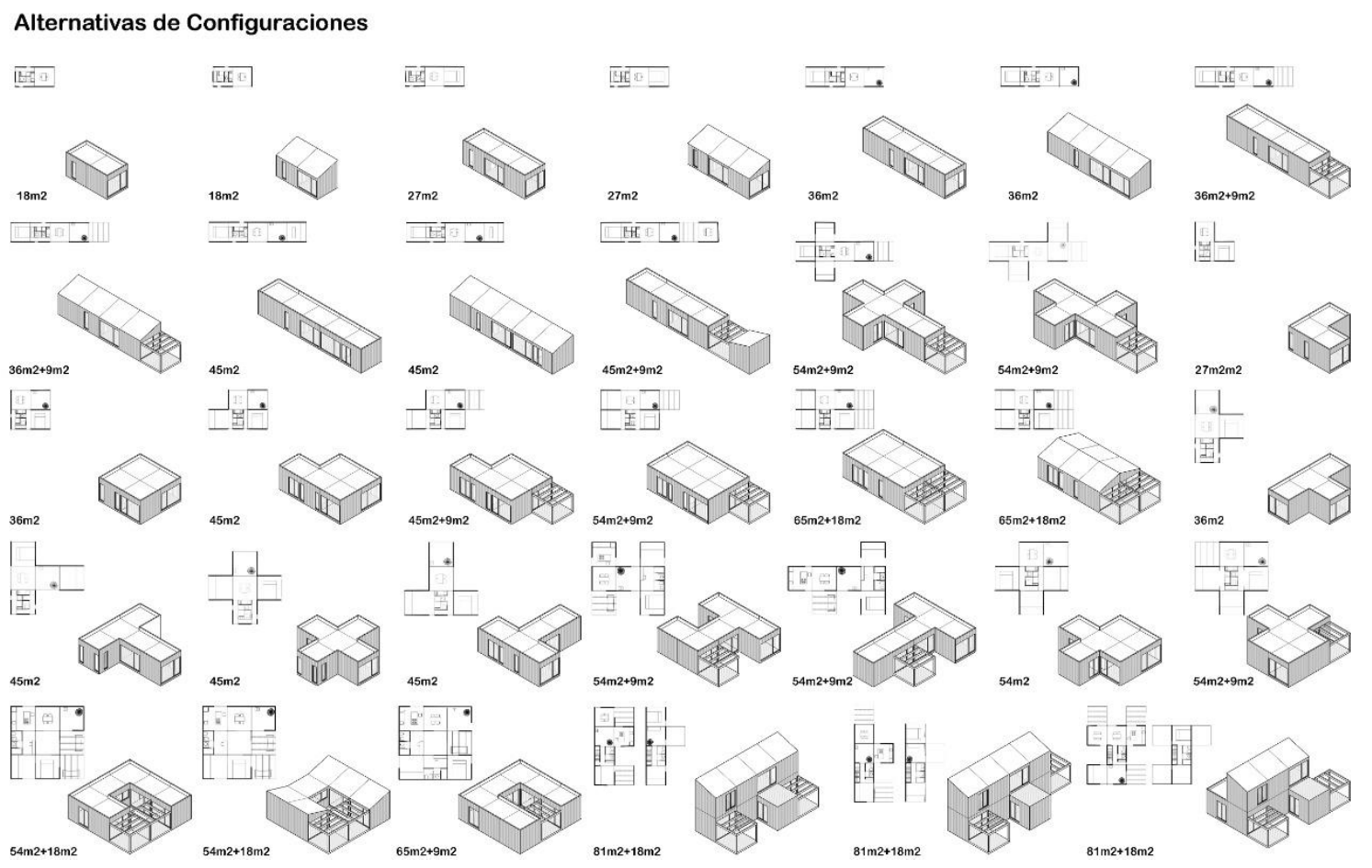

Figura 4: Sistema Incremental Modular. Configuraciones Espaciales Emergentes. Fuente: Elaboración propia 
Este método constructivo se estima que reduce los costos un $40 \%$ respecto del método tradicional. En tanto se construye en un entorno cerrado, es decir, la planta industrial, los obreros no tienen que trasladarse, no hay inclemencias climáticas y las casas se fabrican en "serie" por mano de obra especializada en cada rubro o dentro de la industria 4.0. Por otro lado, al momento de concretar la compra, se define un precio fijo. Así, el efecto de la inflación que suele afectar la construcción tradicional en nuestro país puede ser contemplado en el valor final del proyecto, al efectuar la compra. Por último, dado que las soluciones habitacionales se construyen en poco tiempo, también se evita en ciertos grupos destinatarios pagar expensas e impuestos del terreno que se adquirió para construir, sin estar habitando allí.

Como ventajas adicionales de su practicidad se puede mencionar que la solución habitacional adoptada puede ampliarse con el tiempo, a partir de la incorporación de nuevos módulos que se ensamblan a los existentes mediante la modalidad plug in o conectable. De esta manera, el sistema de tipo incremental posibilita la ejecución por etapas, comenzando por construir un módulo pequeño de 18 metros cuadrados y que luego vaya creciendo, hasta alcanzar una vivienda de 90 metros cuadrados o más.

El proceso de construcción se resume en cuatro fases: (1) Elección de la Configuración espacial elegida para la vivienda (2) Producción de la estructura y envolvente (3) Instalaciones y acabados, (4) Transporte en camiones o sistema de transporte alternativo y (5) Montaje en el lugar con grúa. En este sentido, el modulo tiene dimensiones atendiendo a su máximo ancho (3,00 mtrs), largo (3,00 mtrs) y alto (3,00 - 3,80 mtrs) para que el transporte en el país se pueda hacer en camiones sin el uso de escolta.

SIM pretende abordar la necesidad de soluciones habitacionales considerando la sustentabilidad tanto desde su espectro social, económico, como ambiental. Desde lo social, a partir de la versatilidad de adaptación a cada usuario -caracterizados en la fundamentación-, sus modos de habitar, necesidades y requerimientos; desde lo económico, procurando la maximización de los espacios habitables a un costo accesible; y desde lo ambiental, a partir de la maximización de los recursos utilizados, reduciendo el consumo energético, reutilizando y reciclando el agua de lluvia y grises para su posterior uso en baños, lavado y riego exterior.

Por otra parte, el modelo de sustentabilidad utilizado apunta a generar consciencia ambiental y se encuentra en la base del sistema constructivo empleado, el cual permite una utilización óptima de los recursos: el uso de materiales de construcción tradicionales como la madera, el acero, el hormigón, el yeso de revestimiento (opcional), el agua y el vidrio aislante se reduce al mínimo. Tampoco hay excavación de cimientos en el terreno, dado que el montaje se realiza sobre pilotes de apoyo o zapata corrida sin contacto con el suelo, evitando afectar la naturaleza.

La aislación de la envolvente de enchapados (madera, plástico reciclado o chapa) con espuma de poliuretano expandido o techos verdes en algunas de las materialidades posibles de utilizar, le brinda una óptima temperatura de confort. La espuma de poliuretano, es el material con mayor poder de aislación disponible en el mercado. Además, está incluida en la categoría A dentro de la escala de eficiencia térmica, según la ley de eficiencia energética promulgada recientemente y que obliga a los arquitectos a informar la categoría de aislación térmica de la construcción, que es una oblea igual a la que viene pegada en freezers y heladeras.

Estos sistemas Haus Panel (chapa) y SIP tipo TAO o similares (Madera) de muros panelizados, son una novedad tecnológica en el campo de la construcción. La eficiencia energética y la ingeniería en prefabricación dan como resultado a un elemento constructivo listo para su montaje. En sólo $10 \mathrm{~cm}$ de espesor de muro, piso y techo, se aísla térmicamente lo mismo que un muro de ladrillo macizo de $90 \mathrm{~cm}$. Se reducen costos por calefacción y refrigeración por toda la vida útil de su vivienda.

Por otra parte, SIM-SH incorpora un sistema de climatización pasiva, al tener aberturas en el piso y en el techo, creando un sistema de ventilación natural y aprovechando el efecto termosifón, 
Estrategias Proyectuales para un Hábitat Emergente e Inclusivo

Sistema Incremental Modular para Situaciones Habitacionales

Etulain, Lancioni, Di Gilio, Di Gregorio Luchetti. Estudiantes: Saab, Weber

haciendo que el aire caliente salga por la parte superior del techo y el aire fresco que entra debajo de la casa está en flujo continuo, proporcionando una gran sensación térmica.

En el caso del Hormigón prefabricado, queda directamente expuesto al exterior sin ningún acabado adicional, siendo opcional la incorporación de distintas capas de terminación tanto interior como exterior. Cada módulo actúa al mismo tiempo como elementos de estructura, aislamiento y revestimiento. Esta característica es útil en términos de reducción de cantidades de material, mano de obra, y por lo tanto de costo.

Estos aspectos técnicos, se complementan con la utilización de paneles fotovoltaicos para captar energía solar reduciendo el consumo energético de red, la reutilización y el reciclaje del agua de lluvia y grises para su posterior uso en baños, lavado y riego exterior, al pasar a través de una estación de tratamiento aeróbico y biológico por medio de un sistema de biodigestores trifásicos para finalmente regresar al ambiente en condiciones de pureza física, química y bacteriológica.

Se entiende que la propuesta posibilita la opción de ser implementada en cualquier Región Bioclimática a partir de ir combinando los componentes técnicos adecuados. Como sistema abierto e incremental, SIM es capaz de mutar en el tiempo generando su propia alimentación y minimizando la importación de energías externas, además de no contaminar el ambiente sino más bien procurando mejorar el paisaje natural o urbano, construyendo un hábitat adecuado para el desarrollo humano. (Ver Figura 5)

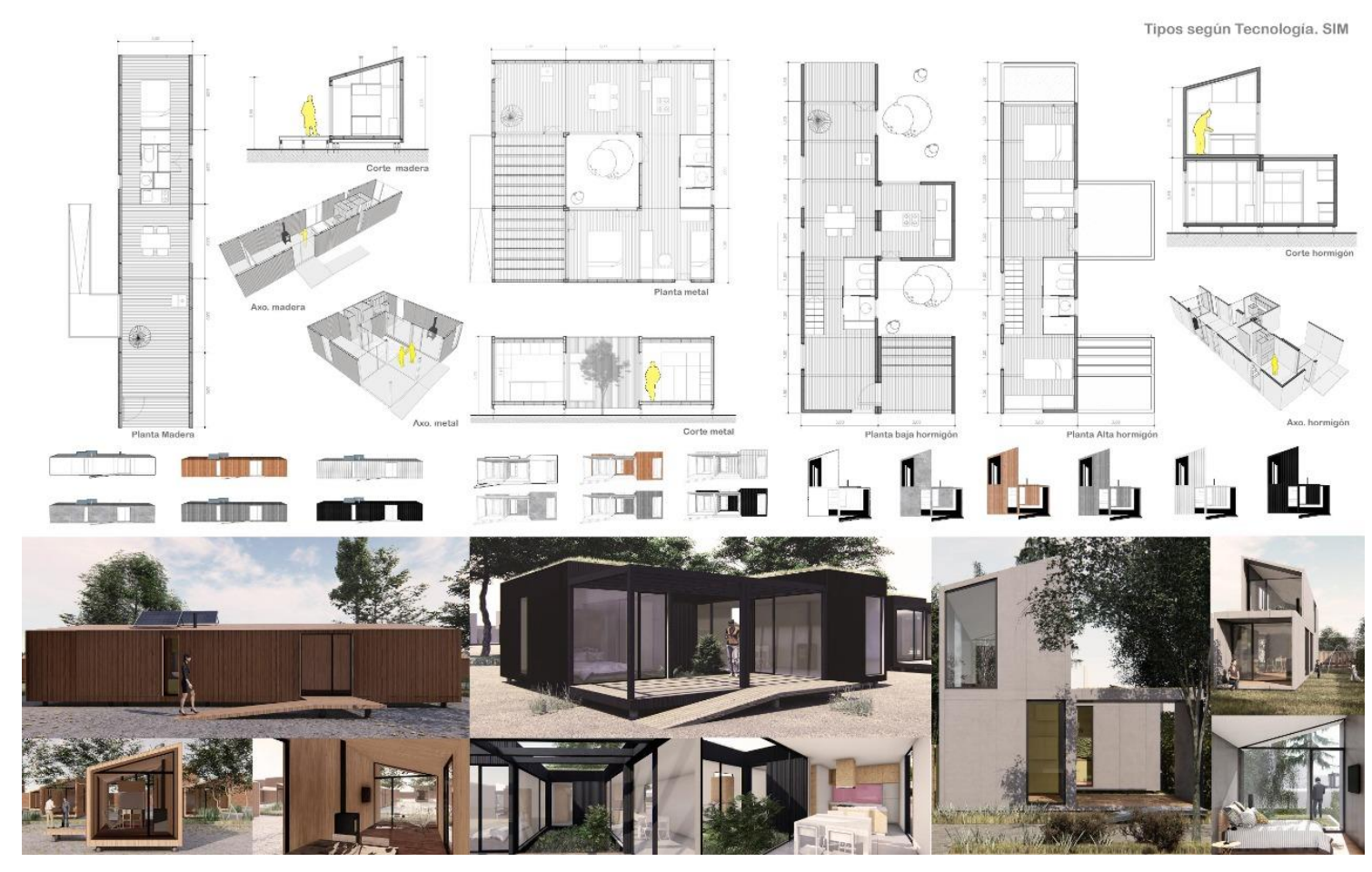

Figura 5: Sistema Incremental Modular. Configuraciones Tipos según Materialidad. Fuente: Elaboración propia 
Estrategias Proyectuales para un Hábitat Emergente e Inclusivo

Sistema Incremental Modular para Situaciones Habitacionales

Etulain, Lancioni, Di Gilio, Di Gregorio Luchetti. Estudiantes: Saab, Weber

\section{OBSERVACIONES FINALES}

Como reflexión final, se considera que el trabajo presentado permite dar respuestas a necesidades emergentes de los sectores más desprotegidos de la sociedad. Desde la perspectiva del riesgo, la población argentina y latinoamericana como se ha expresado, está expuesta a múltiples eventos peligrosos: socio-naturales, sociales y tecnológicos, que la hacen vulnerables y que requieren desde el Estado políticas inclusivas que de manera interdisciplinaria atiendan esta problemática, la cual ha quedado expuesta con toda su crudeza por la pandemia generada a partir del Covid-19. En este contexto, se entiende que tanto la Arquitectura como el Urbanismo como disciplinas que abordan la configuración del espacio de manera holística, tienen mucho para aportar en este sentido.

\section{BIBLIOGRAFÍA}

Por la naturaleza del artículo, se presentan los referentes utilizados:

\section{Arquitectura Modular}

Estudio Borrachia. (2020). Módulo de Emergencia Comunitario (EMC). Argentina. Recuperado de https://arqa.com/arquitectura/modulo-de-emergencia-comunitario.html

Renato Vital. (2020). Madi Home. Italia. Recuperado de https://madihome.com/?utm medium=website\&utm source=plataformaarquitectura.cl

Carballo-Errasti Arquitectos. (2016). Competencia Una Casa - Habitar Contemporánea. Argentina. Recuperado de https://www.carballoerrasti.com/proyectos/una-casa/

\section{Arquitectura en Madera}

Mapa arquitectura. (2016). Sistema MINIMO. Brasil / Uruguay. Recuperado de https://mapaarq.com/

Bio Architects. (2016). DublDom 2.65. Rusia. Recuperado de https://dubldom.com/eu

Estudio Borrachia. (2017). Modulo MECANO. Argentina. Recuperado de https://www.estudioborrachia.com/

Estudio Borrachia. (2019). Hábitat 7. Modulo Transportable para ecoturismo. IGEO. Argentina. Recuperado de https://www.estudioborrachia.com/

Gruba. (2020). Módulos Habitables. Argentina. Recuperado de https://www.gruba.com.ar/objetos-habitables

Baag. (2016). Competencia Una Casa - Habitar Contemporánea. Sistema Fragmentación. Argentina. Recuperado de http://baag.com.ar/producciones/es/26/casa-calu

Casas Cubik. Chile. Recuperado de https://casascubik.cl/

Abaton Arquitectura. (2018). APH80. Casa Prefabricada Móvil. España. Recuperado de https://ecoinventos.com/aph80/

Ivihomes. (2016). Módulos de madera premiun de pino impregnada.Argentina. Recuperado de https://www.ivihomes.com/

\section{Arquitectura Acero}

Mapa arquitectura. (2016). Sistema MINIMO. Brasil / Uruguay. Recuperado de https://mapaarq.com/

Estudio Die Brucke. (2016). Heer Viviendas Modulables. Arquitectura Patagónica. Argentina. Recuperado de https://www.heerviviendasmodulares.com/ 
Estrategias Proyectuales para un Hábitat Emergente e Inclusivo

Sistema Incremental Modular para Situaciones Habitacionales

Etulain, Lancioni, Di Gilio, Di Gregorio Luchetti. Estudiantes: Saab, Weber

Jean Nouvel. (2016). Simple. Francia. Recuperado de https://magaceen.com/es/architecture/simple-by-jean-nouvel/

WE Architecture. (2018). Refugio Gimme, Dinamarca. Recuperado de https://www.we-a.dk/

Arquitectos SysHaus. (2015). WaterlilliHaus. Brasil. Recuperado de http://www.sys.haus/

Moduli. (2014) Casas Modulares Transportable. Argentina. Recuperado de http://www.moduli.com.ar

Box Rental Construcción Modular. (2012). Viviendas Transportables BR. Argentina. Recuperado de http://www.boxrental.com.ar/viviendas-transportables.php

Casa Suite Box. (2015). Arquitectura Modular Sostenible. Argentina. Recuperado de https://www.suitebox.com.ar/

Torrado Arqtos. Place House. Sistema de construcción modular eficiente. Argentina. Recuperado de https://www.instagram.com/place houses/ y https://place.ar/

Idero Arquitectura. (2014). Módulos habitacionales Premium. Argentina. Recuperado de https://www.ideroarquitectura.com/

Grupo Edisur. (2014). Caps Modulares.Argentina. Recuperado de https://steelplex.com.ar/

PLUG Arquitectura (2018). Casas Industrializadas Transportables y Modulares. Argentina. Recuperado de https://www.plugarq.com/

\section{Arquitectura en Hormigón}

Summary Studio. (2019). Viviendas Modulares Vale de Camba. Portugal. Recuperado de http://summary.pt/

Klotz + Deteco. (2020). Casa Prefabricada. Chile. Recuperado de https://www.instagram.com/p/CAy9ZeznL1c/

Schmadke + Grupo Grandio. (2019). Huga. Vivienda Modular. Argentina. Recuperado de https://www.plataformaarquitectura.cl/cl/950360/vivienda-huga-grandio

Hans Zwimpfer + ZAPCO. (2006). Sistema Pile Up. Suiza. Recuperado de https://www.plataformaarquitectura.cl/cl/02-3102/tipologia-arquitectonica-pile-up-zapco

Smartliving MP (2005). Módulos de Hormigón. España. Recuperado de https://www.smartliving.cat/en/contruction-system-en/ 